\title{
Thermodynamic Geometry and Critical Behavior of Black Holes
}

\author{
Jianyong Shen ${ }^{1}$, Rong-Gen $\mathrm{Cai}^{*, 2}$, Bin Wang ${ }^{\dagger, 1}$ and Ru-Keng $\mathrm{Su}^{\ddagger}, 3,1$ \\ ${ }^{1}$ Department of Physics, Fudan University, Shanghai 200433, China \\ ${ }^{2}$ Institute of Theoretical Physics, Chinese Academy of Sciences, \\ P.O. Box 2735, Beijing 100080, China \\ 3 China Center of Advanced Science and Technology (World \\ Laboratory) P.O. Box 8730, Beijing 100080, China
}

\begin{abstract}
Based on the observations that there exists an analogy between the Reissner-Nordström-anti-de Sitter (RN-AdS) black holes and the van der Waals-Maxwell liquid-gas system, in which a correspondence of variables is $(\phi, q) \leftrightarrow(V, P)$, we study the Ruppeiner geometry, defined as Hessian matrix of black hole entropy with respect to the internal energy (not the mass) of black hole and electric potential (angular velocity), for the RN, Kerr and RN-AdS black holes. It is found that the geometry is curved and the scalar curvature goes to negative infinity at the Davies' phase transition point for the RN and Kerr black holes. Our result for the RN-AdS black holes is also in good agreement with the one about phase transition and its critical behavior in the literature.

PACS numbers: 04.70.Dy, 04.65.+e, 05.40.-a
\end{abstract}

\footnotetext{
* Email address: cairg@itp.ac.cn

† Email address: wangb@fudan.edu.cn

${ }^{\ddagger}$ Email address: rksu@fudan.ac.cn
} 


\section{INTRODUCTION}

One of important characteristics of a black hole is its thermodynamic property: a black hole has Hawking temperature proportional to its surface gravity on the horizon of the black hole, entropy proportional to its horizon area 1, 2], and they satisfy the first law of black hole thermodynamics 3], although the statistical origin of the black hole entropy still remains obscure. In general relativity, the most general stationary black hole solution with asymptotical flatness is the KerrNewman solution, which describes a rotating, charged black hole with only three parameters: mass, electric charge and angular momentum. This characteristic is called no hair theorem of black holes. For the Schwarzschild black hole (static, spherically symmetric black hole without electric charge), its Hawking temperature is inversely proportional to its mass, the heat capacity of the black hole is therefore always negative and the black hole is thermodynamically unstable. However, for the Reissner-Nordström (RN) black hole (static, spherically symmetric black hole with electric charge), Kerr black hole (rotating black hole without electric charge), and more general Kerr-Newman black hole, their heat capacity is positive in some parameter region and negative in other region, and between them, the heat capacity diverges. As one knows, the divergence of heat capacity is the indication of a second order phase transition in the ordinary thermodynamic systems. Just on the basis of the divergence of heat capacity, Davies 4] argued that phase transition appears in black hole thermodynamics and the phase transition point is the one where the heat capacity diverges (see also [5]). Some authors investigated different aspects of this critical point [6] and found that some critical exponents related to this critical point obey the scaling laws. On the other hand, some people argued that there exists a critical point at the extremal limit of black holes and a second order phase transition takes place from an extremal black hole to its non-extremal counterpart, some critical exponents related to this critical point also satisfy scaling laws []].

Over the past decade, due to the AdS/CFT correspondence (for a review see [8]), there has been a lot of interest in the thermodynamics of various black holes in anti-de Sitter(AdS) space. It was convincingly argued by Witten [9] that the thermodynamics of black holes in AdS space can be identified with that of the dual conformal field theory (CFT) residing on the boundary of the AdS space. Therefore by studying thermodynamics and phase structure of black holes in AdS space, one can gain sone insights into corresponding ones of dual strong coupling CFTs and vice versa. For instance, the Hawking-Page phase transition [10] between the large stable Schwarzschild black hole phase and thermal AdS space phase can be explained as the deconfinement/confinement phase transition in the dual gauge field theory. In addition, Chamblin et al. 11, 12] investigated 
thermodynamics of RN black holes in AdS space, and revealed rich phase structure of RN-AdS black holes in the fixed charge ensemble. In the spirit of AdS/CFT correspondence, the charge carried by the RN-AdS black hole can be explained as the background current to which the dual CFT couples. The phase structure of RN-AdS black holes is in good agreement with expectation from the dual CFT. In particular, we would like to stress here that the point where the heat capacity diverges with fixed charge for the RN-AdS black hole is indeed a critical point of a second order phase transition. Obviously, the results given by Chamblin et al. also supports the viewpoint of Davies that the divergence point of heat capacity of black holes is a phase transition point, since according to the holographic principle that a theory with gravity can be dual to a theory without gravity in one dimension fewer [13], the thermodynamics of black holes in asymptotically flat spacetime can also be identified with that of a dual theory without gravity, although we do not know so far what the dual theory is.

On the other hand, one can introduce some standard geometrical ideas in ordinary thermodynamics (for a review see 14]). Weinhold [15] was the first to introduce the geometrical concept into the thermodynamics, he suggested a sort of Riemannian metric defined as the second derivatives of internal energy $U$ with respect to entropy and other extensive quantities of a thermodynamic system. However, the geometry based on this metric seems physically meaningless in the context of purely equilibrium thermodynamics. Later soon, Ruppeiner [16] introduced a metric, defined as the second derivatives of entropy $S$ with respect to the internal energy and other extensive quantities of a thermodynamic system. It turns out that the Ruppeiner metric is conformally related to the Weinhold metric with the inverse temperature as the conformal factor. The Ruppeiner geometry has its physically meanings in the fluctuation theory of equilibrium thermodynamics. The components of the inverse Ruppeiner metric gives second moments of fluctuations. Since the proposal of Ruppeiner, many investigations have been carried out on the physically meanings of the Ruppeiner geometry in various thermodynamic systems such as the ideal classical gas, multicomponent ideal gas, ideal quantum gas, one-dimensional Ising model, van der Waals model and so on. In particular, it was found that the Ruppeiner geometry carry information of phase structure of thermodynamic system; and scalar curvature of the metric diverges (goes to negative infinity) at the phase transition and critical point, which shows a strong correlation of system. For thermodynamic systems with no statistical mechanical interactions (for example, ideal gas), the scalar curvature is zero and the Ruppeiner metric is flat. These have been summarized in the review paper [14] (some recent works on the Ruppeiner geometry see 17]).

Since the Ruppeiner geometry in some sense can reveal some features of statistical mechanical 
models, it is therefore of great interest to apply the Ruppeiner geometry to black hole thermodynamics, because so far we do not know their statistical models behind thermodynamics of most black holes, except for a few black holes in superstring/M theories. Indeed, the geometric approach to thermodynamics was first to be introduced to the black hole thermodynamics and to discuss the critical behavior in moduli space by Ferrara et al. [18]. In particular, it was found that the Weinhold metric is proportional to the metric on the moduli space for supersymmetric extremal black holes, whose Hawking temperature is zero, and the Ruppeiner metric governing fluctuations naively diverges, which is consistent with the argument that near extreme the thermodynamic description breaks down [19]. The geometric approach has also been used to the BTZ black hole 20] and RN black holes and Kerr black holes in diverse dimensions [21, 22]. The authors of [21, 22] found that considering the entropy as a function of the mass and charge (angular momentum)of $\mathrm{RN}$ (Kerr) black hole, the Ruppeiner metric is always flat (curved) for the RN (Kerr) black holes and the scalar curvature vanishes for the RN black hole, while it diverges at the extremal Kerr black hole. Namely, the Ruppeiner metric for the RN black holes is quite different from that of the Kerr black holes. In addition there is no special occurring at the phase transition point of Davies.

Based on the observations made by Chamblin et al. [11, 12] and by $\mathrm{Wu}$ [23] for the phase structure of RN-AdS black holes, in the present paper we will reconsider the thermodynamic geometry of RN, Kerr and RN-AdS black holes in four dimensions. Generalization to other higher dimensions is straightforward. One of important observations in [11, 12] is that the phase structure of RN-AdS black holes is analogous to that of the van der Waals-Maxwell liquid-gas system, maybe it is beyond just an analogy. The phase diagram $(Q-T$ diagram, where $Q$ and $T$ denote the charge and Hawking temperature ) of the RN-AdS black holes is quite similar to the phase diagram $(P-T$ diagram, where $P$ and $T$ stand for the pressure and temperature ) of the van-der Waals-Maxwell system; the equation of state $Q-\phi$ diagram (where $\phi$ denotes the electric potential on the horizon of black hole) of the RN-AdS black holes is similar to that of $P-V$ diagram ( $V$ is the volume) of the van der Waals-Maxwell system. That is, in this analogy of the RN-AdS black hole to the van der Waals-Maxwell system, the electric potential $\phi$ plays the role of the extensive quantity volume $V$ and the charge $Q$ the role of the intensive quantity pressure $P$. In addition, let us notice that an appropriate order parameter is the difference of the electric potentials in two phases in the RN-AdS black holes, while the order parameter is the difference of energy densities between liquid phase and gas phase in the van der Walls-Maxwell system. Furthermore, we redefine the internal energy $U$ of black holes ( the ADM gravitational mass of black holes subtracted by the energy of electric field outside the black hole horizon (for charged black holes) and/or the energy due to the rotation 
(for the rotating black holes). Considering the entropy of black holes as a function of the internal energy and electric potential (angular velocity) of RN, Kerr and RN-AdS black holes, we obtain the Ruppeiner metric and calculate the scalar curvature of geometry. We find that the result is consistent with the expectation: the scalar curvature diverges at the phase transition points of Davies for the RN and Kerr black holes; the case of the RN-AdS black holes is also in complete agreement with [11, 12, 23].

This paper is organized as follows. In Sec. II and Sec. III, we will respectively discuss the thermodynamic geometry of the RN black hole and Kerr black hole, and calculate their scalar

curvature $\hat{R}$, which indeed goes to negative infinity at the phase transition point of Davies. In Sec. IV, we will first briefly review the thermodynamics of the RN-AdS black hole and then study its thermodynamic geometry. We end this paper with conclusion and discussions in Sec. V.

\section{THERMODYNAMIC GEOMETRY OF RN BLACK HOLES}

The charged static black hole is known as the RN black hole whose metric is given by

$$
d s^{2}=-f(r) d t^{2}+f(r)^{-1} d r^{2}+r^{2} d \Omega^{2}
$$

in four dimensional spacetime, where $d \Omega^{2}$ is the line element on a unit 2-sphere and

$$
f(r)=1-\frac{\mu}{r}+\frac{q^{2}}{r^{2}}
$$

$\mu / 2=M$ and $q$ is the ADM mass and electric charge of the black hole respectively in units ( $G=c=\hbar=k_{B}=1$ ) which we use throughout this paper. The two horizons of the RN black hole, inner Cauchy horizon located at $r_{-}$and outer event horizon at $r_{+}$, can be expressed by mass and electric charge

$$
\mu=r_{-}+r_{+}, \quad q^{2}=r_{-} r_{+}
$$

with the condition $q^{2} \leq \mu^{2} / 4$ ruling out the naked singularity at $r=0$. When $r_{-}$equals to $r_{+}$, the black hole is extremal. The entropy of the black hole is obtained by the area law

$$
S=A / 4=\pi r_{+}^{2}
$$

The energy conservation law of the black hole

$$
d M=T d S+\phi d q
$$


implies the Hawking temperature $T$ and electric potential on the event of horizon $\phi$ of the RN black hole

$$
\begin{gathered}
T=\left(\frac{\partial M}{\partial S}\right)_{q}=\frac{r_{+}-r_{-}}{4 \pi r_{+}^{2}}, \\
\phi=\left(\frac{\partial M}{\partial q}\right)_{S}=\frac{\sqrt{r_{+} r_{-}}}{r_{+}}=\frac{q}{r_{+}} .
\end{gathered}
$$

The combination of Eq.(3), Eq.(6) and Eq.(17) provides an equation of state (EOS) of the RN black hole $\phi=\phi(q, T)$.

Before proceeding, let us discuss in some detail what roles play by $\phi$ and $q$ in the analogy of the RN-AdS black hole to the van der Waals-Maxwell system, for the latter, the first law of thermodynamics is

$$
d u=T d S-P d V
$$

where $u$ is the internal energy, $P$ and $V$ are respectively the pressure and volume of the system. As presented in the Sec. IV (also see 11, 12, 23]), although the electrical charge $q$ looks like an extensive variable and the potential $\phi$ like an intensive one, from the isotherms in the $q-\phi$ plane (see Fig. 1) and the $q-T$ phase diagram of the RN-AdS black hole, we can see that $\phi$ and $q$, respectively, play the roles of volume $V$ and pressure $P$ in the corresponding diagrams of the van der Waals-Maxwell system. That is, the correspondence is $(\phi, q) \rightarrow(V, P)$ for establishing the phase structure of the RN-AdS black holes. The internal energy represents the basic and intrinsic properties of the thermodynamic system, which excludes the contribution of the external work. For the existence of the RN black hole, the intrinsic property is obviously determined by the structure of the spacetime itself excluding the effects of the static electricity. Therefore the appropriate internal energy should be understandably written as

$$
u=M-\phi q .
$$

Expressed by the internal energy, the first law of thermodynamics is written as

$$
d u=T d S-q d \phi
$$

Comparing (8) and (10), we can clearly establish the correspondence $(\phi, q) \rightarrow(V, P)$. Other pieces of supporting evidence to express the internal energy of charged black hole as (9) come as follows. When expressing the entropy of the RN-AdS black holes in terms of the Cardy-Verlinde formula, 
we noticed that the energy of electric field outside the black hole horizon has to be subtracted from the mass $M$ of the black hole [24]; For a rotating body, its internal energy is the difference between the total energy of the body and its kinetic energy of rotation [25]. It is therefore reasonable to argue that the discussion above for the RN-AdS black hole also holds for the asymptotically flat RN and Kerr black holes. That is, the internal energy of the RN black hole should be defined as in (91). As a state function, entropy should be a function of internal energy.

We now turn to the thermodynamic geometry of the RN black hole. The thermodynamic metric introduced in Ruppeiner's theory [14] is defined by the second derivatives of the entropy. It is worth paying attention that the entropy inducing the metric must be in the strict form of the function regarded as the internal energy and the extensive variables in ordinary thermodynamic systems

$$
\hat{g}_{a b}=\frac{\partial^{2}}{\partial x^{a} \partial x^{b}} S(x) \quad(a, b=1,2, \ldots, n)
$$

where $x=\left(u, x^{1}, x^{2}, \ldots, x^{n-1}\right)$ denotes the internal energy $u$ and the extensive variables $x^{a} \quad(a \neq 1)$. Based on the discussions above, for the RN black hole, the thermodynamic metric can be written down as

$$
\hat{g}_{a b}=\frac{\partial^{2}}{\partial x^{a} \partial x^{b}} S(u, \phi) \quad(a, b=1,2)
$$

where $x^{1}=u$ and $x^{2}=\phi$. Note that for the van der Waals model, the entropy is a function of the internal energy $u$ and fluid density $\rho$ [14]. Using Eq.(4), the direct calculation yields

$$
\hat{g}=\left(\begin{array}{cc}
-\frac{4 \pi r_{+}^{2} \sqrt{r_{+} r_{-}}}{r_{+}\left(r_{+}-r_{-}\right)^{2}} & \frac{2 \pi r_{+}^{2}}{\left(r_{+}-r_{-}\right)^{2}} \\
\frac{2 \pi r_{+}^{2}}{\left(r_{+}-r_{-}\right)^{2}} & \frac{\pi r_{+}^{3}\left(r_{+}+5 r_{-}\right)}{\left(r_{+}-r_{-}\right)^{2}}
\end{array}\right)
$$

and the scalar curvature is

$$
\hat{R}=\hat{g}_{a b} \hat{R}^{a b}=-\frac{r_{+}-r_{-}}{\pi r_{+}\left(3 r_{-}-r_{+}\right)^{2}} .
$$

We see in our setup that the scalar curvature vanishes only at the extremal limit where $r_{+}=r_{-}$. In a general case, the scalar curvature does not vanish and it goes to negative infinity when $r_{+}=3 r_{-}$, which stands for a kind of phase transition or long range correlation of the system according to the Ruppeiner's theory [14]. It is interesting to note that the divergence point of the scalar curvature is just the phase transition point of Davies [4]. It is easy to check this by calculating the heat capacity with a fixed charge

$$
C_{q}=T\left(\frac{\partial S}{\partial T}\right)_{q}=\frac{2 \pi r_{+}^{2}\left(r_{+}-r_{-}\right)}{3 r_{-}-r_{+}}
$$


which is singular at $3 r_{-}=r_{+}$and indicates that the black hole has a second order phase transition 4, 6]. Therefore, the Ruppeiner's theory well describes the critical behavior of RN black hole thermodynamics as it does in ordinary thermodynamic systems, after carefully understanding of some quantities of black hole thermodynamics.

\section{THERMODYNAMIC GEOMETRY OF KERR BLACK HOLES}

The rotating black hole without charge is known as Kerr black hole, whose line element is

$$
\begin{aligned}
d s^{2}= & -\frac{\Delta-a^{2} \sin ^{2} \theta}{\Sigma} d t^{2}-\frac{2 a \sin ^{2} \theta\left(r^{2}+a^{2}-\Delta\right)}{\Sigma} d t d \phi+\frac{\left(r^{2}+a^{2}\right)^{2}-\Delta a^{2} \sin ^{2} \theta}{\Sigma} \sin ^{2} \theta d \phi^{2} \\
& +\frac{\Sigma}{\Delta} d r^{2}+\Sigma d \theta^{2},
\end{aligned}
$$

where

$$
\Sigma=r^{2}+a^{2} \cos ^{2} \theta \quad \Delta=r^{2}+a^{2}-\mu r .
$$

Here $\mu / 2=M$ and $J=a M=a \mu / 2$ are the ADM mass and the angular momentum of the Kerr black hole respectively. Both the outer and inner horizons $\left(r_{+}\right.$and $\left.r_{-}\right)$are given by $\Delta=$ $r^{2}+a^{2}-\mu r=0$ and have the relation

$$
\mu=r_{+}+r_{-} \quad J=a M=\frac{1}{2} \sqrt{r_{+} r_{-}}\left(r_{+}+r_{-}\right)
$$

By the area law, the entropy of the Kerr black hole is

$$
S=\frac{1}{4} A=\pi\left(r_{+}^{2}+a^{2}\right)=\pi\left(r_{+}^{2}+r_{+} r_{-}\right) .
$$

According to the energy conservation law $d M=T d S+\Omega_{H} d J$, the Hawking temperature and the angular velocity of the outer horizon are respectively

$$
T=\left(\frac{\partial M}{\partial S}\right)_{J}=\frac{r_{+}-r_{-}}{4 \pi\left(r_{+}^{2}+r_{+} r_{-}\right)}
$$

and

$$
\Omega_{H}=\left(\frac{\partial M}{\partial J}\right)_{S}=\frac{\sqrt{r_{+} r_{-}}}{2\left(r_{+}^{2}+r_{+} r_{-}\right)} .
$$

Just like the case in the RN black hole, we are able to establish thermodynamics of the Kerr black hole by using the correspondence $\left(\Omega_{H}, J\right) \rightarrow(V, P)$. The internal energy of the Kerr black hole should exclude the kinetic energy of rotation, that is

$$
u=M-\Omega_{H} J
$$


and the first law of thermodynamics can be written down as

$$
d u=T d S-J d \Omega_{H}
$$

By the definition of the thermodynamic metric in Eq.(11), for the Kerr case, we have

$$
\hat{g}_{a b}=\frac{\partial^{2}}{\partial x^{a} \partial x^{b}} S\left(u, \Omega_{H}\right),
$$

where $a, b=1,2$ and $x^{1}=u, x^{2}=\Omega_{H}$. A simple calculation gives

$$
\hat{g}=\left(\begin{array}{cc}
\frac{2 \pi\left(r_{+}+r_{-}\right)^{3}}{\left(r_{+}-r_{-}\right)^{3}} & \frac{8 \pi r_{+}^{2} \sqrt{r_{+} r_{-}}\left(r_{+}+r_{-}\right)^{2}}{\left(r_{+}-r_{-}\right)^{3}} \\
\frac{8 \pi r_{+}^{2} \sqrt{r_{+} r_{-}}\left(r_{+}+r_{-}\right)^{2}}{\left(r_{+}-r_{-}\right)^{3}} & \frac{2 \pi r_{+}^{2}\left(r_{+}+r_{-}\right)^{3}\left(r_{+}^{2}+6 r_{+} r_{-}-r_{-}^{2}\right)}{\left(r_{+}-r_{-}\right)^{3}}
\end{array}\right) .
$$

The scalar curvature of the Kerr black hole is

$$
\hat{R}=\hat{g}_{a b} \hat{R}^{a b}=-\frac{4\left(r_{+}+r_{-}\right)\left(r_{+}^{2}-9 r_{-}^{2}\right)}{\pi\left(r_{+}-r_{-}\right)\left(r_{+}^{2}-6 r_{+} r_{-}-r_{-}^{2}\right)^{2}} .
$$

We see that $\hat{R}$ naively diverges at the extreme limit of the Kerr black hole where $r_{+}=r_{-}$, which is of less physically interest since at the extremal limit the Hawking temperature vanishes, the thermodynamics description breaks down as mentioned above. An interesting divergence of $\hat{R}$ for a non-extremal black hole occurs at $r_{+}^{2}-6 r_{+} r_{-}-r_{-}^{2}=0$, at which the heat capacity with a fixed angular momentum

$$
C_{J}=-\frac{2 \pi r_{+}\left(r_{+}-r_{-}\right)\left(r_{+}+r_{-}\right)^{2}}{r_{+}^{2}-6 r_{+} r_{-}-r_{-}^{2}}
$$

also becomes singular and stands for thermodynamic critical phenomena. Again this is also consistent with the phase transition point of Kerr black hole given by Davies [4]. In addition, let us note that the scalar curvature vanishes at $r_{+}=3 r_{-}$, namely, $a=3 M / 4$. At the moment we do dot know whether there are any special physical meanings by the vanishing of the scalar curvature at this point.

\section{THERMODYNAMIC GEOMETRY OF RN-ADS BLACK HOLES}

Compared to the RN and Kerr black holes, the RN-AdS black holes have much more rich thermodynamic characteristics including phase transition and critical behavior [11, 12, 23], which also have good agreements in the frame of the thermodynamic geometry. The metric of the RN-AdS black hole in four dimensions is

$$
d s^{2}=-f(r) d t^{2}+f(r)^{-1} d r^{2}+r^{2} d \Omega^{2}
$$


where

$$
f(r)=1-\frac{\mu}{r}+\frac{q^{2}}{r^{2}}+\frac{r^{2}}{l^{2}}
$$

with the negative cosmological constant $\Lambda=-3 / l^{2}$. The gravitational mass $M=\mu / 2$ and the electric charge $q$ are given by the inner and outer horizons

$$
\begin{gathered}
\mu=r_{+}+r_{-}+\frac{r_{+}^{4}-r_{-}^{4}}{l^{2}\left(r_{+}-r_{-}\right)}, \\
q^{2}=r_{+} r_{-}\left(1+\frac{r_{+}^{3}-r_{-}^{3}}{l^{2}\left(r_{+}-r_{-}\right)}\right) .
\end{gathered}
$$

The black hole entropy $S$ is

$$
S=\frac{1}{4} A=\pi r_{+}^{2}
$$

Through the energy conservation law $d M=T d S+\phi d q$, we have the Hawking temperature and electric potential on the horizon of the black hole

$$
T=\left(\frac{\partial M}{\partial S}\right)_{q}=\frac{\left(r_{+}-r_{-}\right)\left(l^{2}+3 r_{+}^{2}+2 r_{+} r_{-}+r_{-}^{2}\right)}{4 \pi l^{2} r_{+}^{2}}
$$

and

$$
\phi=\left(\frac{\partial M}{\partial q}\right)_{S}=\frac{\sqrt{r_{+} r_{-}\left[1+\left(r_{+}^{2}+r_{+} r_{-}+r_{-}^{2}\right) / l^{2}\right]}}{r_{+}}=\frac{q}{r_{+}} .
$$

The EOS of the RN-AdS black hole $q=q(\phi, T)$ can be obtained by using Eq. (30)-Eq. (334) and thus the isotherm can be drawn on the $q-\phi$ state plane as shown in Fig 1 We can see indeed that the isotherms on the $q-\phi$ state plane is quite similar to those on the $P-V$ diagram of the van der Waals model. We note that when $T>T_{c}$, there are a locally maximum point and a locally minimum point, both are determined by

$$
\left(\frac{\partial q}{\partial \phi}\right)_{T}=\frac{r_{+}\left(3 r_{+}^{4}-l^{2} r_{+}^{2}+3 l^{2} q^{2}\right)}{2\left(3 r_{+}^{4}-l^{2} r_{+}^{2}+l^{2} q^{2}\right)}=0 .
$$

The isotherm can be divided into three branches in this stage as suggested in 11]. "Branch 3 " is the branch which extends all the way from $q=\infty$ and terminates at $d q / d \phi=0$. This branch is electrically stable and has negative free energy in the its most part. "Branch 2" covers the branch between the locally maximum and minimum points, where there is an instability by thermal fluctuations like the case of Van de Waals' gas 11, 12], in spite of its electrical stability. 


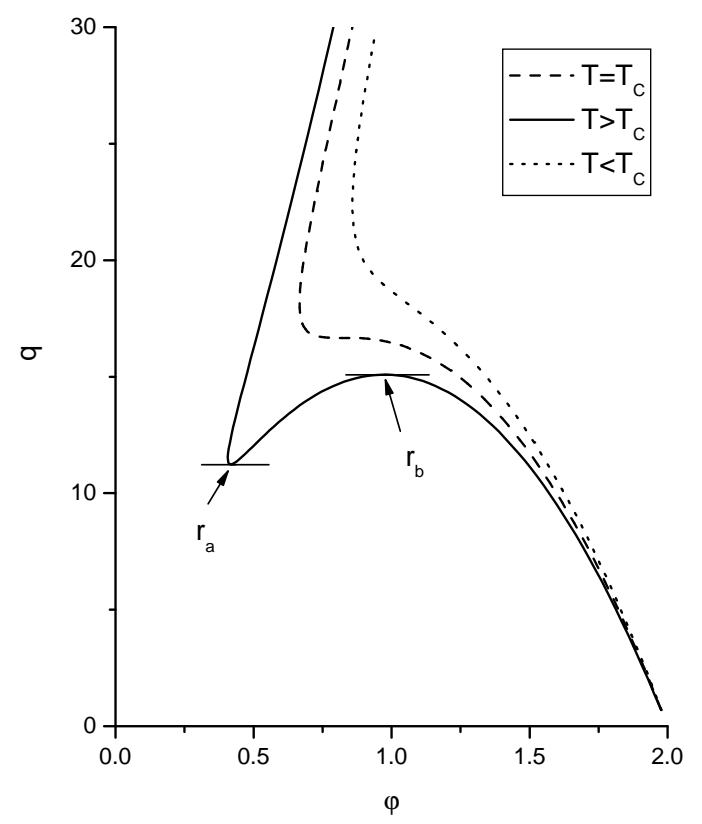

Figure 1: The EOS of the RN-AdS black hole when the temperature $T$ is equal to, less and greater than the critical temperature $T_{c}$ respectively. $r_{a}$ and $r_{b}$ are the values of $r_{+}$when $q$ reaches the local minimum and maximum, which corresponds to the divergences of $C_{q}$.

The rest branch is called "Branch 1". Hence, we still can use the "equal area law" to get the first order phase transition. The heat capacity for a fixed charge is

$$
\begin{aligned}
C_{q} & =T\left(\frac{\partial S}{\partial T}\right)_{q}=\frac{2 \pi r_{+}^{2}\left(r_{+}-r_{-}\right)\left(l^{2}+3 r_{+}^{2}+2 r_{+} r_{-}+r_{-}^{2}\right)}{3 r_{+}^{3}-l^{2} r_{+}+3 r_{-}\left(l^{2}+r_{+}^{2}+r_{+} r_{-}+r_{-}^{2}\right)} \\
& =\frac{2 \pi r_{+}^{3}\left(r_{+}-r_{-}\right)\left(l^{2}+3 r_{+}^{2}+2 r_{+} r_{-}+r_{-}^{2}\right)}{3 r_{+}^{4}-l^{2} r_{+}^{2}+3 l^{2} q^{2}}
\end{aligned}
$$

and it has singularities at $3 r_{+}^{4}-l^{2} r_{+}^{2}+3 l^{2} q^{2}=0$, whose roots are

$$
r_{a}=\left(\frac{l}{6}\left(1-\sqrt{1-\frac{36 q^{2}}{l^{2}}}\right)\right)^{1 / 2}, \quad r_{b}=\left(\frac{l}{6}\left(1+\sqrt{1-\frac{36 q^{2}}{l^{2}}}\right)\right)^{1 / 2}
$$

which correspond to the local maximum and minimum in Fig 1. The states between these two points on the isotherm of $T>T_{c}$, i.e., $r_{a}<r_{+}<r_{b}$, stand on the Branch 2 and are unstable. When $T=T_{c}$, the Branch 2 shrinks to one critical point

$$
\left(\frac{\partial q}{\partial \phi}\right)_{T}=\left(\frac{\partial^{2} q}{\partial \phi^{2}}\right)_{T}=0
$$




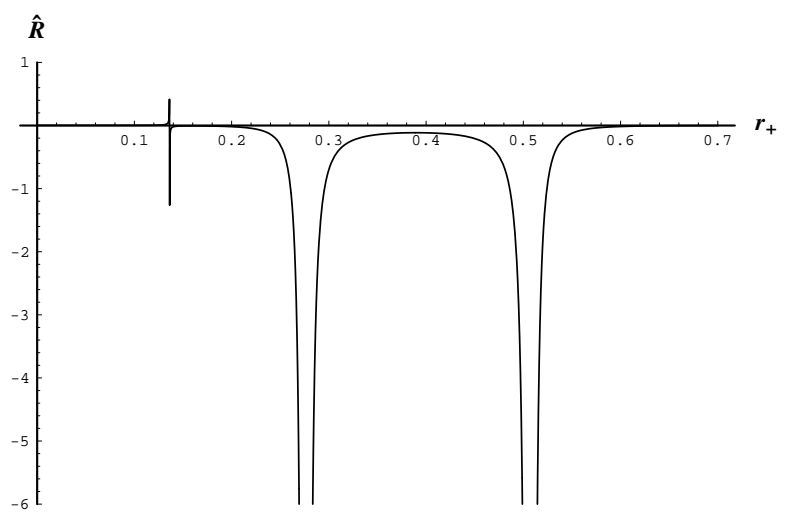

Figure 2: The thermodynamic scalar curvature $\hat{R}$ of the RN-AdS black hole vs. the outer event horizon for fixed electric charge $q<q_{c}$.

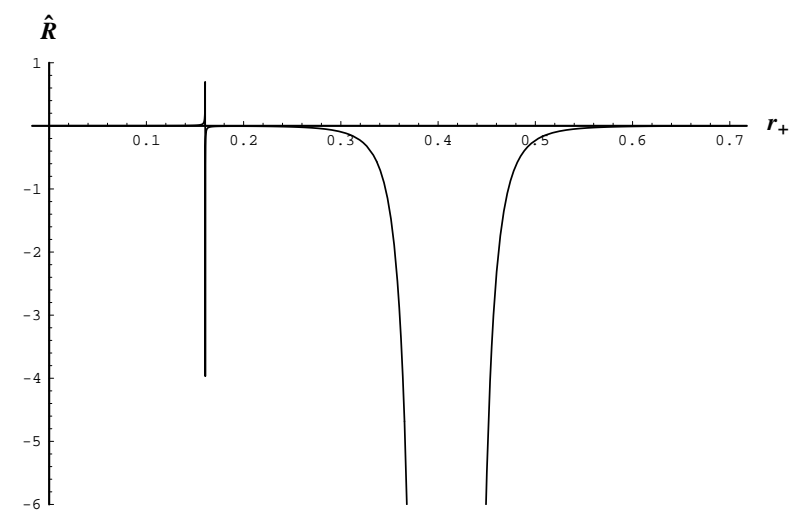

Figure 3: The thermodynamic scalar curvature $\hat{R}$ of the RN-AdS black hole vs. the outer event horizon for fixed electric charge $q=q_{c}$.

where the second order phase transition happens and $r_{a}=r_{b}$ with $r_{c}^{2}=l^{2} / 6, q_{c}^{2}=l^{2} / 36$. Below the critical temperature $T_{c}$, the Branch 2 vanishes and there is a transition of electrical stability at $d q / d \phi=\infty$ between Branch 3 and Branch 1 .

The thermodynamics and phase structure of the RN-AdS black holes have been investigated in details in 11, 12, 23]. All results are consistent with the expectation from the dual field theory via holography. We will not reproduce those results here, instead we just stress that Fig [ suggests a correspondence of variables with $(\phi, q) \rightarrow(V, P)$ so as to establish the analogy of RN-AdS black hole with the van der Waals model. As the cases of RN and Kerr black holes, the internal energy of the black hole system should reflect the intrinsic properties of the spacetime. Entropy should be a function of the internal energy of system. Therefore, the internal energy of the RN-AdS black hole is

$$
u=M-\phi q
$$




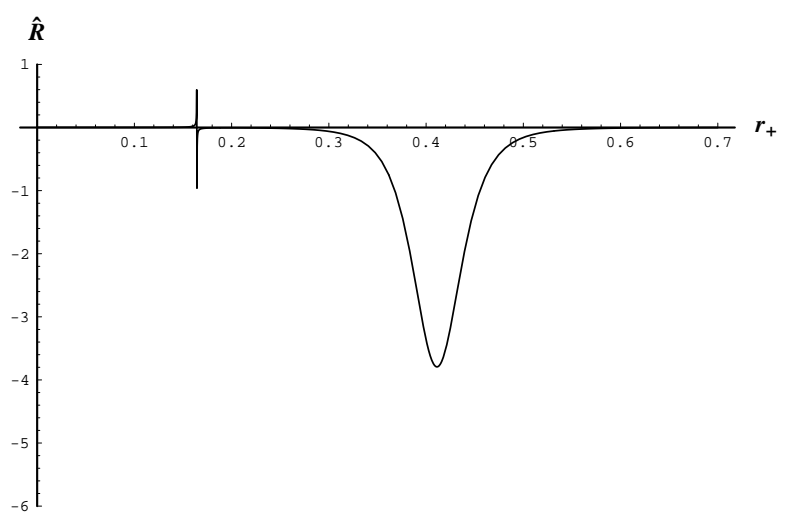

Figure 4: The thermodynamic scalar curvature $\hat{R}$ of the RN-AdS black hole vs. the outer event horizon for fixed electric charge $q>q_{c}$.

and the first law of thermodynamics is

$$
d u=T d S-q d \phi
$$

Through the definition of the metric of thermodynamic metric Eq.(12), we can obtain the scalar curvature of the thermodynamic geometry

$$
\hat{R} \propto-\left\{\left(r_{+}-r_{-}\right)\left(l^{2}+3 r_{+}^{2}+2 r_{+} r_{-}+r_{-}^{2}\right)\left(3 r_{+}^{4}-l^{2} r_{+}^{2}+3 l^{2} q^{2}\right)^{2}\right\}^{-1}
$$

which is rather complicated and listed in detail in the Appendix. Fig 24 show the behavior of $\hat{R}$ for the various fixed electrical charge $q$. The very narrow vertical line is caused by the singularity of the extremal black hole, whose temperature is zero and this divergence reflects the breaking down of thermodynamic description for the extremal black holes [18]. Of interest is the case with non-vanishing Hawking temperature. We see from Fig. 2 that when $q<q_{c}$, the scalar curvature $\hat{R}$ has two negative infinities where $C_{q}$ diverges and $d q / d \phi=0$. A second order phase transition occurs when these two infinities merge into one in the case of $q=q_{c}$ at $r_{a}=r_{b}=r_{c}$ in Fig 3, which indicates the appearance of a long range correlation in the system. If $q>q_{c}$ as shown in Fig 4 , there is no singular point for $\hat{R}$ except the extremal state of the black hole.

In order to see the thermodynamic behavior near the critical point, the critical exponents can be introduced as

$$
\begin{array}{ll}
\text { (1) } & q-q_{c} \sim\left|\phi-\phi_{c}\right|^{\delta} \quad\left(T=T_{c}\right), \\
\text { (2) } & \phi-\phi_{c} \sim\left|T-T_{c}\right|^{\beta} \quad\left(q=q_{c}\right), \\
\text { (3) } & C_{q} \sim\left|T-T_{c}\right|^{-\alpha} \quad\left(q=q_{c}\right), \\
\text { (4) } \quad \kappa_{T} \sim\left|T-T_{c}\right|^{-\gamma} & \left(q=q_{c}\right) .
\end{array}
$$


According to the Ref. [23], $\delta=3, \beta=1 / 3, \alpha=2 / 3$ and $\gamma=2 / 3$ for the RN-AdS black hole and they obey the scaling symmetry like ordinary thermodynamic systems

$$
\begin{aligned}
& \alpha+2 \beta+\gamma=2 \\
& \alpha+\beta(\delta+1)=2 \\
& \gamma(\delta+1)=(2-\alpha)(\delta-1) \\
& \gamma=\beta(\delta-1)
\end{aligned}
$$

On the other side, the thermodynamic scalar curvature $\hat{R}$ is proportional to $\left|T-T_{c}\right|^{\alpha-2}$ near the critical point. Ruppeiner [14] pointed out that the multiplication $R C_{p} t^{2}$ should be a universal constant related to the critical exponents $\beta$ and $\delta[14]$, that is

$$
R C_{p} t^{2}=-\beta(\delta-1)(\beta \delta-1) k_{B}
$$

where $t=\left|T-T_{c}\right| / T_{c}$. The value of the r.h.s of Eq.(44) is zero if $\delta=3$ and $\beta=1 / 3$ are taken. In the case of the RN-AdS black hole, our calculation shows that $R C_{q} t^{2}$ is still a non-zero constant, which implies the difference between thermodynamics of an ordinary system and of a black hole. This is not surprising because there are some differences between black holes and ordinary thermodynamic systems after all. For instance, black holes can have negative heat capacity, entropy of black holes is not an extensive quantity and so on.

\section{CONCLUSION AND DISCUSSION}

The Ruppeiner metric, defined as the Hessian matrix of entropy with respect to internal energy and other extensive variables of a thermodynamic system, is closely related to the fluctuation theory of equilibrium thermodynamics. It was argued that the Riemannian geometry of the Ruppeiner metric can give insights into the underlying statical mechanical system. In particular, it was shown that the scalar curvature of the Ruppeiner geometry carries much information on the phase structure of the thermodynamic system and it diverges at critical points. Therefore it is quite interesting to apply the geometry approach to black hole thermodynamics.

The authors of Refs. 21, 22] defined the Ruppeiner metric of RN (Kerr) black hole as the second derivatives of black hole entropy with respect to the black hole mass and electric charge (angular momentum). It was found that the Ruppeiner geometry is flat and the scalar curvature vanishes for the RN black holes, while it is curved and scalar curvature diverges at the extremal limit for the Kerr black holes. Clearly it is easy to see that a statistical model without any interaction cannot 
re-produce thermodynamic properties of the RN black hole. Furthermore, let us notice that for the RN and Kerr black holes, their thermodynamic properties are quite similar. So it is not easy to understand the results of [21, 22]. In this paper, we have re-investigated the Ruppeiner geometry for the RN, Kerr and RN-AdS black holes, based on the observations for an analogy between the RN-AdS black hole and the Van der Waals-Maxwell liquid-gas system. According to the analogy, an interesting correspondence is $(q, \phi) \rightarrow(P, V)$, as we explained above (also see [11, 12, 23] for details), although it looks strange that the electric potential $\phi$ plays the role of the extensive volume $V$. Based on this observation, we are enforced to think that the black hole mass does not stand for the internal energy as in an ordinary thermodynamic system. As a thermodynamic system, the internal energy of a black hole should be the difference between the mass of black hole and the energy of electric field for the RN black hole (the kinetic energy due to rotation for the Kerr black hole), see (9) and (22), respectively. Considering the entropy of black hole as a function of internal energy and electric potential for charged black holes (angular velocity for rotating black holes), we have calculated the scalar curvature of resulting Ruppeiner metric for the RN, Kerr and RN-AdS black holes. We have found that the Ruppeiner geometry is curved and the scalar curvature goes to negative infinity at the phase transition points of Davies for the RN and Kerr black holes, where heat capacity with a fixed charge (angular momentum) diverges. In the case of non-extremal RN-AdS black hole, the thermodynamic scalar curvature has two singularities when the electrical charge $q$ is below its critical value $q_{c}$, which indicates thermodynamic instability and the first order phase transition as in Van de Waals liquid-gas system. The mergence of these two singularities implies the existence of the second order phase transition of the black hole at $q=q_{c}$. The black hole behaves like a gas system with interaction for $q>q_{c}$. Our results are completely consistent with those in Refs. [11, 12, 23].

We have also investigated the critical behavior of the scalar curvature $\hat{R}$ and found that the scaling symmetry $R C_{q} t^{2}$ is still a non-zero constant, not as that of the ordinary thermodynamic system predicated in Eq. (44). These results indicate that while the Ruppeiner's theory does work well and is consistent with the classical thermodynamics even in the system of black holes, there are some differences between the thermodynamics of black holes and that of ordinary thermodynamic system. For example, as it was noticed in [23] that critical exponents do not match each other for the RN-AdS black hole and the van der Waals model, although their phase structures are quite similar. In addition, although the divergence of scalar curvature of the Ruppeiner geometry is in good agreement with the black hole phase transition in our setup, it is clearly needed to further understand the physical meanings of the Ruppeiner geometry in the black hole thermodynamics. 


\section{APPENDIX}

In this appendix we give the Ruppeiner metric and its scalar curvature for the RN-AdS black holes. We calculate the thermodynamic metric as the function of inner and outer horizons. Hence, the Jacobi matrix must be used here

$$
J=\left(\begin{array}{cc}
r_{+, u} & r_{+, \phi} \\
r_{-, u} & r_{-, \phi}
\end{array}\right)=\left(\begin{array}{ll}
u_{, r_{+}} & u_{, r_{-}} \\
\phi_{, r_{+}} & \phi_{, r_{-}}
\end{array}\right)^{-1} .
$$

Using the definition of the thermodynamic metric Eq.(12) and Jacobi Eq.(45), we obtain

$$
\hat{g}=\left(\begin{array}{cc}
S_{, u u} & S_{, u \phi} \\
S_{, \phi u} & S_{, \phi \phi}
\end{array}\right),
$$

where

$$
\begin{aligned}
& S_{, u u}=\frac{2 \pi l^{2} r_{+}\left(3 r_{+}^{4}-l^{2} r_{+}^{2}+q^{2} l^{2}\right)}{\left(r_{+}-r_{-}\right)^{3}\left(l^{2}+3 r_{+}^{2}+2 r_{+} r_{-}+r_{-}^{2}\right)^{3}}, \\
& S_{, u \phi}=S_{, \phi u}=\frac{4 \pi l^{2} r_{+} q\left(l^{2} r_{+}^{2}-q^{2} l^{2}\right)}{\left(r_{+}-r_{-}\right)^{3}\left(l^{2}+3 r_{+}^{2}+2 r_{+} r_{-}+r_{-}^{2}\right)^{3}}, \\
& S_{, \phi \phi}=\frac{\pi l^{2} r_{+}^{3} z\left(r_{+}, r_{-}\right)}{\left(r_{+}-r_{-}\right)^{3}\left(l^{2}+3 r_{+}^{2}+2 r_{+} r_{-}+r_{-}^{2}\right)^{3}},
\end{aligned}
$$

and

$$
\begin{aligned}
z\left(r_{+}, r_{-}\right)= & 9 r_{+}^{6}+r_{+}^{4}\left(6 l^{2}-5 r_{-}^{2}\right)-5 r_{-}^{2}\left(l^{2}+r_{-}^{2}\right)^{2}+2 r_{+}^{3}\left(2 l^{2} r_{-}-5 r_{-}^{3}\right) \\
& +r_{+}^{2}\left(l^{4}-6 l^{2} r_{-}^{2}-15 r_{-}^{4}\right)+2 r_{+}\left(2 l^{4} r_{-}-3 l^{2} r_{-}^{3}-5 r_{-}^{5}\right)
\end{aligned}
$$

After a tedious calculation, finally we have the scalar curvature

$$
\hat{R}=-\frac{B\left(r_{+}, r_{-}\right)}{\pi\left(r_{+}-r_{-}\right)\left(l^{2}+3 r_{+}^{2}+2 r_{+} r_{-}+r_{-}^{2}\right)\left(3 r_{+}^{4}-l^{2} r_{+}^{2}+3 q^{2} l^{2}\right)^{2}},
$$

where

$$
\begin{aligned}
B\left(r_{+}, r_{-}\right)= & 54 r_{+}^{7} r_{-}+l^{2} r_{-}^{2}\left(l^{2}+r_{-}^{2}\right)^{2}+r_{+}^{6}\left(-18 l^{2}+84 r_{-}^{2}\right)+3 r_{+}^{5}\left(9 l^{2} r_{-}+38 r_{-}^{3}\right) \\
& +r_{+}^{4}\left(9 l^{4}+34 l^{2} r_{-}^{2}+90 r_{-}^{4}\right)+r_{+}^{3}\left(-29 l^{4} r_{-}+35 l^{2} r_{-}^{3}+60 r_{-}^{5}\right) \\
& +r_{+}\left(-2 l^{6} r_{-}+25 l^{2} r_{-}^{5}\right)+r_{+}^{2}\left(l^{6}+30 l^{4} r_{-}^{2}+63 l^{2} r_{-}^{4}+30 r_{-}^{6}\right) .
\end{aligned}
$$

It is easy to check that $\hat{R}$ holds the form of Eq.(14) when the limit $l \rightarrow \infty$ is taken. 


\section{Acknowledgments}

RGC thanks X.S. Chen and H.Lt Shi for useful discussions. This research was initiated when RGC was visiting the department of physics, Fudan university, whose hospitality extends to him is grateful. This work was supported in part by NNSF of China, by the National Basic Research Program 2003CB716300 and the Foundation of Education of Ministry of China. B. Wang's work was also supported in part by Shanghai Science and Technology Commission.

[1] S. W. Hawking, Commun. Math. Phys. 43, 199 (1975).

[2] J. D. Bekenstein, Phys. Rev. D 7, 2333 (1973).

[3] J. M. Bardeen, B. Carter and S. W. Hawking, Commun. Math. Phys. 31, 161 (1973).

[4] P. C. W. Davies, Proc. Roy. Soc. Lond. A 353, 499 (1977); P. C. W. Davies, Rep. Prog. Phys. 41, 1313 (1977) P. C. W. Davies, Class. Quant. Grav. 6, 1909 (1989).

[5] P. Hut, Mon. Not. R. Astron. Soc. 180, 379 (1977).

[6] L.M. Sokolowski and P. Mazur, J. Phys. A13, 1113 (180); Y.K. Lau, Phys. Lett. A 186, 41 (1994); Y.K. Lau, Phys. Lett. A188, 245 (1994); J. P. Muniain and D. Piriz, Phys. Rev. D 53, 816 (1996); C.O. Lousto, Nucl. Phys. B410, 433 (1993); C.O. Lousto, Phys. Rev. D51, 1733 (1995).

[7] A. Curir, Gen. Rel. Grav. 13, 417 (1981); A. Curir, Gen. Rel. Grav. 13, 1177 (1981); D. Pavon and M.J. Rubbi, Phys. Rev. D37, 2052 (1988); D. Pavon, Phys. Rev. D43, 2495 (1991); R. G. Cai, R. K. Su and P. K. N. Yu, Phys. Rev. D 48 (1993) 3473; R.K. Su, R.G. Cai and P.K. N. Yu, Phys. Rev. D50, 2932 (1994); O.K. Kaburaki, Phys. Lett. A217,315 (1996); O.K. Kaburaki, Gen. Rel. Grav. 28, 843 (1996); R. G. Cai, Z. J. Lu and Y. Z. Zhang, Phys. Rev. D 55, 853 (1997) arXiv:gr-qc/9702032; R. G. Cai, Phys. Rev. Lett. 78, 2531 (1997) arXiv:hep-th/9702142; R. G. Cai and Y. S. Myung, Nucl. Phys. B 495, 339 (1997) arXiv:hep-th/9702159; R. G. Cai and L. Z. Qiao, Commun. Theor. Phys. 31 (1999) 459; R. G. Cai, J. Korean Phys. Soc. 33, S477 (1998) arXiv:gr-qc/9901026.

[8] O. Aharony, S.S. Guber, J. Maldacena, H. Ooguri and Y. Oz, Phys. Rep. 323, 183 (2000).

[9] E. Witten, Adv. Theor. Math. Phys. 2, 505 (1998) arXiv:hep-th/9803131.

[10] S. W. Hawking and D. N. Page, Commun. Math. Phys. 87, 577 (1983).

[11] A. Chamblin, R. Emparan, C. V. Johnson and R. C. Myers, Phys. Rev. D 60, 064018 (1999) arXiv:hep-th/9902170.

[12] A. Chamblin, R. Emparan, C. V. Johnson and R. C. Myers, Phys. Rev. D 60, 104026 (1999) arXiv:hep-th/9904197.

[13] G. 't Hooft, arXiv:gr-qc/9310026 L. Susskind, J. Math. Phys. 36, 6377 (1995) arXiv:hep-th/9409089.

[14] G. Ruppeiner, Rev. Mod. Phys. 67, 605 (1995), 68, 313 (E) (1996).

[15] F. Weinhold, J. Chem. Phys. 63, 2479 (1975). 
[16] G. Ruppeiner, Phys. Rev. A 20, 1608 (1979).

[17] D. A. Johnston, W. Janke and R. Kenna, Acta Phys. Polon. B 34, 4923 (2003) arXiv:cond-mat/0308316.

[18] S. Ferrara, G. W. Gibbons and R. Kallosh, Nucl. Phys. B 500, 75 (1997) arXiv:hep-th/9702103.

[19] J. Preskill, P. Schwarz, A. D. Shapere, S. Trivedi and F. Wilczek, Mod. Phys. Lett. A 6, 2353 (1991);

R. Kallosh, A. D. Linde, T. Ortin, A. W. Peet and A. Van Proeyen, Phys. Rev. D 46, 5278 (1992) arXiv:hep-th/9205027.

[20] R. G. Cai and J. H. Cho, Phys. Rev. D 60, 067502 (1999) arXiv:hep-th/9803261.

[21] J. Aman, I. Bengtsson and N. Pidokrajt, Gen. Rel. Grav. 35, 1733 (2003) arXiv:gr-qc/0304015.

[22] J. E. Aman and N. Pidokrajt, arXiv:hep-th/0510139

[23] X. N. Wu, Phys. Rev. D 62, 124023 (2000).

[24] R. G. Cai, Phys. Rev. D 63, 124018 (2001) arXiv:hep-th/0102113; R. G. Cai, Y. S. Myung and N. Ohta, Class. Quant. Grav. 18, 5429 (2001) arXiv:hep-th/0105070. R. G. Cai and A. z. Wang, Phys. Rev. D 70, 064013 (2004) arXiv:hep-th/0406057; R. G. Cai, D. W. Pang and A. Wang, Phys. Rev. D 70, 124034 (2004) arXiv:hep-th/0410158.

[25] L.D. Landau and E.M. Lifshitz, Statistical Physics, 3rd Edition, Part 1, \$26 (Pergamon Press,1980). 